\title{
Comportamento ingestivo de vacas de corte submetidas a diferentes níveis nutricionais no terço final da gestação
}

Ingestive behavior of beef cows submitted to different nutritional levels in the final third of pregnancy

Comportamiento ingestivo de vacas de corte sometidas a diferentes niveles nutricionales en el tercero final del gestación

Sander Martinho Adams ORCID: https://orcid.org/0000-0003-4895-8237 Universidade Federal de Santa Maria, Brasil E-mail: sander.adams@ hotmail.com John Lenon Klein ORCID: https://orcid.org/0000-0001-8337-4152 Universidade Federal de Santa Maria, Brasil E-mail: johnlenonklein@gmail.com

Diego Soares Machado ORCID: https://orcid.org/0000-0002-2406-280X Universidade Federal de Santa Maria, Brasil E-mail: dsm_zootecnista@hotmail.com

Leonel da Silva Rodrigues

ORCID: https://orcid.org/0000-0002-9272-9151

Universidade Federal de Santa Maria, Brasil E-mail: rodrigues_leonel@hotmail.com Ivan Luiz Brondani

ORCID: https://orcid.org/0000-0002-6526-3042 Universidade Federal de Santa Maria, Brasil

E-mail: ivanbrondani@gmail.com

Dari Celestino Alves Filho

ORCID: https://orcid.org/0000-0003-2559-7504

Universidade Federal de Santa Maria, Brasil E-mail: darialvesfilho@ hotmail.com

Joziane Michelon Cocco

ORCID: https://orcid.org/0000-0001-6617-3076

Universidade Federal de Santa Maria, Brasil

E-mail: jozimichelon@hotmail.com

Fabiana Moro Maidana

ORCID: https://orcid.org/0000-0002-9946-5355

Universidade Federal de Santa Maria, Brasil

E-mail: f96033512@gmail.com

Gabriele Amaro de Oliveira

ORCID: https://orcid.org/0000-0001-8675-2315

Universidade Federal de Santa Maria, Brasil

E-mail: gabrieleamarodeoliveira@gmail.com

Matheus Dutra Teixeira

ORCID: https://orcid.org/0000-0001-6990-4401

Universidade Federal de Santa Maria, Brasil

E-mail: duttramatheus@gmail.com

Raíssa Lixinski de Souza

ORCID: https://orcid.org/0000-0002-1750-6505

Universidade Federal de Santa Maria, Brasil

E-mail: raissalixinski16@outlook.com

Resumo

O objetivo deste trabalho foi a valiar o comportamento ingestivo de vacas de corte submetidas à diferentes níveis nutricionais no terço final de gestação. Foram utilizadas 18 vacas prenhas das raças Charolês e Nelore, divididas nos tratamentos: Baixo (exclusivamente em pastagem natural); Médio (suplementação para atender as exigências de mantença); Alto (suplementação para atender $150 \%$ das exigências de mantença). O delineamento experimental inteiramento casualizado com seis repetições por tratamento e três períodos de avaliação foi utilizado. O tempo 
destinado a outras atividades aumentou com o avançar da gestação e a medida que o nível nutricional neste período também aumentou, com valores de 0,96; 1,86 e 3,24 horas dia-1 para os tratamentos Baixo, Médio e Alto respectivamente. Vacas do tratamento Alto demonstraram serem mais seletivas, uma vez que apresentaram menor tempo por estação (6,96 vs 9,18 e 10,19 segundos) e percorreram maior número de estações por minuto $(9,51$ vs 7,06 e 7,31) em relação às vacas Baixo e Médio, respectivamente. Menor tempo por bocado (1,45 segundos) e consequentemente maior taxa de bocados $(41,86$ bocados $/ \mathrm{min})$ foi obtido nas vacas Baixo em relação às vacas Médio e Alto. Maior seletividade e eficiência de pastejo ocorreram ao final da gestação, com menor deslocamento entre estações (1,03 passos) e menor tempo em cada estação (6,42 segundos) resultando em maior número de estações/minuto (10,01 estações). Vacas do nível Baixo apresentam maior eficiência na apreensão do pasto, enquanto que vacas suplementadas apresentam maior seletividade. Com o avançar da gestação, vacas aumentam a seletividade do material ingerido para atender maiores demandas metabólicas associadas a menor capacidade de consumo.

Palavras-chave: Estação de pastejo; Pastagem nativa; Ruminação; Suplementação; Tempo de pastejo.

\begin{abstract}
The objective of this work was evaluate the ingestive behavior of beef cows submitted to different nutritional levels in the final third of gestation. Eighteen pregnant cows of the Charolais and Nellore breeds were used, divided into treatments: Low (exclusively on natural pasture); Medium (supplementation to meet maintenance requirements); High (supplementation to meet $150 \%$ of maintenance requirements). The randomized complete experimental design was used with six replicates per treatment and three evaluation periods. The time spent in other activities increased with pregnancy advancement and as the nutritional level in this period also increased, with values of 0.96; 1.86 and 3.24 hours day-1 for the Low, Medium and High treatments respectively. High treatment cows proved to be more selective, since they had less time per station (6.96 vs 9.18 and 10.19 seconds) and went through a higher number of stations per minute $(9.51$ vs 7.06 and 7,31$)$ in relation to the Low and Medium cows, respectively. Shortest time per bite (1.45 seconds) and consequently a greater number of bite rate $(41.86 \mathrm{bites} / \mathrm{min})$ was obtained in the Low cows compared to Medium and High cows. Greater selectivity and grazing efficiency occurred at pregnancy end, with less displacement between stations (1.03 steps) and less time per station (6.42 seconds) resulting in a greater number of stations / minute (10.01 stations). Low-level cows are more efficient in apprehending pasture, while supplemented cows are more selective. As gestation advances, cows increase the selectivity of material ingested to meet greater metabolic demands associated with less consumption capacity.
\end{abstract}

Keywords: Grazing station; Grazing time; Native pasture; Rumination; Supplementation.

\title{
Resumen
}

El objetivo de este trabajo fue evaluar el comportamiento ingestivo de vacas de carne sometidas a diferentes niveles nutricionales en el tercio final de la gestación. Se utilizaron 18 vacas preñadas de las razas Charolais y Nellore, divididas en tratamientos: Bajo (exclusivamente en pasto natural); Medio (suplementación para cumplir con los requisitos de mantenimiento); Alto (suplementación para cubrir el 150\% de los requisitos de mantenimiento). Se utilizó el diseño experimental completo aleatorizado con seis repeticiones por tratamiento y tres períodos de evaluación. El tiempo destinado a otras actividades se incrementó con el avance de la gestación y como también cuando se incrementó el nivel nutricional en este período, con valores de 0,96; 1,86 y 3,24 horas día-1 para los tratamientos Bajo, Medio y Alto respectivamente. Las vacas del tratamiento Alto demostraron ser más selectivas, ya que tuvieron menos tiempo por estación de alimentación (6,96 vs 9,18 y 10,19 segundos) y pasaron por un mayor número de estaciones por minuto $(9,51$ vs 7,06 y 7, 31) en relación a las vacas Bajo y Medio, respectivamente. Se obtuvo menos tiempo por bocado (1.45 segundos) y consecuentemente un mayor número de bocados/minuto (41.86 bocados) en las vacas Bajo en relación a las vacas Medio y Alto. Mayor selectividad y eficiencia de pastoreo ocurrió al final de la gestación, con desplazamiento menor entre estaciones (1.03 pasos) y menos tiempo en cada estación (6.42 segundos) resultando en un mayor número de estaciones/minuto (10.01 estaciones). Las vacas de bajo nivel son más eficientes en la captura del pasto, mientras que las vacas suplementadas son más selectivas. Con el avance de la gestación, las vacas aumentan la selectividad del material ingerido para satisfacer mayores demandas metabólicas asociadas con una menor capacidad de consumo.

Palabras clave: Estación de pastoreo; Pasto nativo; Rumia; Suplementación; Tiempo de pastoreo.

\section{Introdução}

A oscilação no valor nutricional da forragem é uma característica do Bioma Pampa. Essa pastagem possui bom valor nutricional na primavera e no verão e baixa qualidade no outono e inverno (Pereira et al., 2018). A fase mais crítica do rebanho de cria se concentra nos meses que antecedem o parto, sendo comum observar situações onde vacas se encontram em restrição alimentar, com falta de nutrientes para manter desenvolvimento fetal adequado. Bauman e Currie (1980) afirmam que existem mecanismos que definem a formação fetal como prioridade nutricional para as vacas, sendo que estas irão mobilizar reservas 
corporais para atender as demandas placentárias, causando redução na condição corporal da vaca ao parto e consequentemente baixos índices produtivos na estação reprodutiva subsequente.

O sistema de cria é complexo, pois exige maior conhecimento e capacidade administrativa além de possuir alto risco e baixa rentabilidade (Mello et al., 2013). A melhoria na competitividade da pecuária tem início na base da bovinocultura de corte, o sistema de cria, etapa relacionada à reprodução e com a produção de bezerros (Barcellos \& Oaigen, 2014). O uso de técnicas mais intensivas de produção, como a suplementação para complementar a deficiência nutricional das forragens, podem ser eficientes para melhorar o desempenho das vacas gestantes. Bohnert et al. (2013) observaram aumento de 12,3\% na taxa de repetição de prenhes em vacas que pariram com melhor condição corporal em função da suplementação no último trimestre de gestação. Os estudos que avaliam os efeitos da suplementação, ou da melhor condição nutricional das vacas durante a gestação, sobre as características reprodutivas das fêmeas no pós-parto, buscam compreender os efeitos da melhor nutrição materna durante a gestação sobre os efeitos à longo prazo no desenvolvimento da progênie, termo conhecido como programação fetal (Du et al., 2010).

Porém, poucos estudos envolvendo vacas de corte na fase final de gestação buscam estudar os efeitos das técnicas envolvidas em melhores sistemas produtivos, tais como a suplementação, sobre o comportamento ingestivo dos animais, fator que pode ser muito importante nesta fase de produção, uma vez que as vacas possuem maiores exigências nutricionais e menor capacidade de ingestão, devido ao acentuado crescimento fetal nos três últimos meses de gestação, onde segundo Bauman e Currie (1980), ocorre 70\% do crescimento fetal. Maior volume abdominal causado pelo peso do concepto e suas membranas e líquidos fetais, também pode influenciar o comportamento animal, uma vez que pode dificultar o deslocamento e apreensão da forragem pelas vacas, aspecto que pode ser amenizado com o uso do suplemento para satisfazer as exigências de mantença. Logo, o objetivo do presente estudo foi avaliar os efeitos da inclusão da suplementação para atender 100 ou $150 \%$ das exigências nutricionais de vacas de corte no terço final de gestação sobre o comportamento ingestivo e padrão de deslocamento dos animais em pastagem natural.

\section{Metodologia}

O experimento foi desenvolvido no Laboratório de Bovinocultura de Corte do Departamento de Zootecnia da Universidade Federal de Santa Maria, situado no município de Santa Maria, no período de 15 de agosto de 2017 a 15 de maio de 2018. O clima da região é o "Cfa" (subtropical úmido), conforme classificação de Köppen, sendo a precipitação pluviométrica média anual entre 1600 a $1900 \mathrm{~mm}$, temperatura de $18,8^{\circ} \mathrm{C}$, com média mínima de $9,3^{\circ} \mathrm{C}$ e média máxima de $24,7^{\circ} \mathrm{C}$ (Alvares et al., 2013).

\section{Animais e Tratamentos}

Utilizou-se 18 vacas de corte prenhas, as quais foram distribuídas em três tratamentos: Baixo (vacas consumindo pastagem natural); Médio (vacas suplementadas buscando-se atender $100 \%$ das exigências de mantença) e Alto (vacas suplementadas buscando-se atender $150 \%$ das exigências de mantença). As vacas foram aleatoriamente distribuídas de acordo com os fatores genética (CH, NE, 11/16 CH, 11/16 NE, 21/32 CH e 21/32 NE) e idade (4 a 12 anos).

As vacas foram mantidas em quatro piquetes de pastagem natural, com áreas de 20,0; 21,0; 41,0 e 47,0 hectares, com suplementação mineral (ProduBeef 60P) de livre acesso. A massa de forragem foi determinada a cada 14 dias pela técnica da dupla amostragem (Wilm, 1944), objetivando-se manter uma massa de forragem (MF) residual de $3500 \mathrm{~kg}$ de matéria seca ha1, devido a composição grosseira da pastagem natural e a participação de aproximadamente $70 \%$ da espécie exótica capim Annoni (Eragrostis plana Nees). A cada período de 28 dias foram realizados rodízios de piquetes para retirar os efeitos da pastagem. A massa e a oferta de forragem nos piquetes foram respectivamente de $4144.72 \mathrm{~kg}$ de matéria seca ha- 1 e $11.22 \mathrm{~kg}$ 
de matéria seca por $100 \mathrm{~kg}$ de peso vivo animal. A lotação média foi equivalente a $275.21 \mathrm{~kg} \mathrm{ha}^{-1}$ de peso corporal.

A suplementação foi realizada diariamente as 11:00 horas da manhã na ordem de 0,28 e $0,98 \%$ do peso vivo para as vacas Médio e Alto, respectivamente, e perdurou até o momento do parto das fêmeas, totalizando em média 95 dias $( \pm 17$ dias). O suplemento foi considerado um aditivo ao consumo de forragem, o qual foi estimado em 2,1\% do peso vivo de matéria seca, seguindo as recomendações descritas pelo National Research Council (1998). Este plano nutricional exposto na Tabela 1, permitiu ganhos diários de peso na ordem de $-0.103 ; 0.025$ e $0.207 \mathrm{~kg} \mathrm{dia}^{-1}$ durante o terço final de gestação para os tratamentos Baixo, Médio e Alto, respectivamente, resultando em melhor condição corporal destas matrizes ao parto (2.81, 2.92 e 2.99 pontos, na mesma ordem).

Tabela 1 - Composição bromatológica da pastagem, fração concentrada e consumos de nutrientes pelas matrizes no terço final de gestação.

\begin{tabular}{|c|c|c|c|}
\hline \multirow{2}{*}{ Fração da dieta } & \multicolumn{3}{|c|}{ Tratamentos } \\
\hline & Baixo & Médio & Alto \\
\hline \multicolumn{4}{|c|}{ Composição bromatológica da Pastagem } \\
\hline Proteína bruta, $\%$ & 5,45 & 5,45 & 5,45 \\
\hline Nutrientes digestíveis totais, $\%$ & 33,89 & 33,89 & 33,89 \\
\hline \multicolumn{4}{|c|}{ Composição bromatológica da fração concentrada } \\
\hline Proteína bruta, $\%$ & - & 18.00 & 15.00 \\
\hline Nutrientes digestíveis totais, $\%$ & - & 85.00 & 85.00 \\
\hline \multicolumn{4}{|c|}{ Consumo de matéria seca e nutrientes para vacas gestantes com $475 \mathrm{~kg}$ de peso vivo } \\
\hline Forragem nativa, $\mathrm{kg} \mathrm{dia}^{-1}$ & 9.98 & 9.98 & 9.98 \\
\hline Suplemento concentrado, $\mathrm{kg} \mathrm{dia}^{-1}$ & - & 1.32 & 4.69 \\
\hline Nutrientes digestíveis totais $\mathrm{kg} \mathrm{dia}^{-1}$ & 4.69 & 5.81 & 8.60 \\
\hline Proteína bruta, $\mathrm{kg} \mathrm{dia}^{-1}$ & 0.45 & 0.70 & 1.15 \\
\hline Nutrientes digestíveis totais, $\%{ }^{1}$ & 88.50 & 109.60 & 162.30 \\
\hline Proteína bruta, $\%^{1}$ & 60.00 & 93.40 & 153.30 \\
\hline
\end{tabular}

Composição da forragem nativa: Proteína bruta 4,5\%; Nutrientes digestíveis totais 47,0\%; Fibra em detergente neutro $80 \%$ (Silveira et al., 2014);

${ }^{1}$ Consumo em relação às exigências diárias de nutrientes digestíveis totais $(5,30 \mathrm{~kg})$ e de proteína bruta $(0,75 \mathrm{~kg})$.

Fonte: Autores.

\section{Avaliações}

As avalições do comportamento ingestivo foram realizadas a cada 28 dias (22/Agosto; 20/Setembro e 17/Outubro) em 6 vacas de cada tratamento durante 12 horas (7:00 as 19:00 horas). A cada 10 minutos foram monitoradas as atividades de pastejo (horas/dia), outras atividades (horas/dia) e ruminação (horas/dia), em que, o tempo de pastejo é considerado o período no qual ocorrerá a prática de apreensão da forragem pelo animal, incluindo pequenos deslocamentos. O período em que o animal estava mastigando o bolo alimentar e não pastejando foi considerado como tempo de ruminação. O período que o animal não estava pastejando, tampouco ruminando ou realizando atividades sociais como frequentar cochos de suplementação mineral e de ingestão de água foi considerado como tempo de outras atividades.

As atividades referentes à apreensão, ingestão e ruminação da forragem pelos animais também foram mensuradas. Para avaliar o padrão de ruminação dos animais foi aferido o tempo e o número de mastigadas por bolo alimentar regurgitado. Durante as atividades de pastejo, foram quantificados o tempo e o número de passos para os animais percorrerem 10 estações 
de pastejo, seguindo a metodologia descrita por Laca et al. (1992), sendo realizado o cálculo do número de passos e estações alimentares realizadas por minuto. A taxa de bocados foi mensurada seguindo a metodologia de Hodgson et al. (1982), pela quantificação do tempo necessário para o animal realizar 20 bocados, sendo posteriormente calculado o número de bocados por minuto. Posteriormente, utilizou-se o tempo de pastejo diurno para calcular o número de passos, estações alimentares e bocados realizados pelo animal durante o dia.

\section{Análise estatística}

Foi utilizado o delineamento experimental inteiramente casualizado, com três tratamentos e três períodos de avaliações. O teste de Shapiro-Wilk foi realizado para testar a normalidade dos resíduos, e transformações realizadas quando necessário. Os dados foram submetidos à análise de variância pelo teste F, através do procedimento PROC MIXED, para as medidas repetidas no tempo avaliadas a cada período experimental. Quando constatada significância na análise de variância, foi realizado o teste de comparação de médias (TCM) pelo teste de Tukey à nível de 5\% de probabilidade, sendo as médias comparadas pelo método dos quadrados mínimos. Todas as análises foram realizadas utilizando o pacote estatístico SAS® (Statistical Analysis System, versão 9.4), seguindo o seguinte modelo matemático:

$$
\mathrm{Y}_{i j k}=\mu+\mathrm{N}_{i}+\mathrm{P}_{j}+(\mathrm{N} * \mathrm{P})_{i j}+\operatorname{Rep}_{k}\left(\mathrm{~N}_{j}\right)+\varepsilon_{i j k}
$$

Onde: $\gamma_{i j k}$ : variáveis dependentes; $\mu$ : média de todas as observações; $\mathrm{N}_{i}$ : efeito do $i$-ésimo nível nutricional pré-parto; $\mathrm{P}_{j}$ : efeito do $j$-ésimo período; $(\mathrm{N} * \mathrm{P})_{i j}$ : efeito da $i j$-ésima interação entre tratamento e período; Rep $k$ : efeito da $k$-ésima repetição dentro dos níveis nutricionais; $\varepsilon_{i j k}$ : efeito do erro aleatório residual (erro b).

\section{Resultados}

A interação entre o tempo de pastejo e período de avaliação comportamental está apresentada na Tabela 2. A interação entre estes dois fatores foi fortemente influenciada pelas vacas Médio e Alto, as quais devido à suplementação apresentaram os menores tempos de pastejo, principalmente no primeiro período de avaliação (mês de agosto), onde a pastagem possui baixa qualidade nutricional. Com o avançar da gestação, o tempo de pastejo foi aumentando nas matrizes suplementadas, enquanto que nas vacas do tratamento Baixo, ocorreu redução no tempo de ingestão de forragem de 10,11 para 9,16 horas, caracterizando a interação para esta característica comportamental.

\section{Nível nutricional no terço final de gestação}

O tempo destinado a outras atividades foi maior conforme aumentou o nível de suplementação das matrizes (Tabela 3), com menor valor nas vacas Baixo $\left(0,96 ; 1,86\right.$ e 3,24 horas dia $\left.^{-1}\right)$. As atividades referentes ao tempo ruminação dia ${ }^{-1}$ dos animais, número de mastigadas e tempo de ruminação por bolo ruminal não foram influenciados pelos tratamentos, demonstrando que a inclusão do suplemento não altera o padrão de ruminação.

Os níveis nutricionais no terço final de gestação não influenciaram o padrão de deslocamento das vacas durante o pastejo, com valores semelhantes para o número de passos por estação alimentar, passos por minuto e passos por dia. Porém, vacas Alto permaneceram menos tempo por estação alimentar (6,96 vs 9,18 e 10,19 segundos) e percorreram maior número de estações por minuto (9,51 vs 7,06 e 7,31 estações alimentares) em relação às vacas Baixo e Médio, respectivamente. Apesar deste resultado, o número de estações frequentadas pelos animais ao longo do dia (3920) não foi influenciado pelos tratamentos.

$\mathrm{Na}$ avaliação da apreensão de forragem (Tabela 3), vacas do Baixo apresentaram menor tempo por bocado (1,45 vs 1,64 e 1,72 segundos) e consequentemente maior número de bocados por minuto (41,86 vs 37,37 e 35,39 bocados) quando comparadas respectivamente com as fêmeas Médio e Alto. A maior eficiência na apreensão e ingestão da forragem pelas vacas 
com menor nível nutricional (Baixo), repercutiu em maior número de bocados realizados por estes animais ao longo do dia ( $\mathrm{P}$ $=0,0009)$. O número de bocados dia ${ }^{-1}$ reduziu à medida que aumentou o nível nutricional das vacas durante o terço final de gestação, com valores respectivamente de 23119, 18693 e 14811 bocados para as vacas Baixo, Médio e Alto.

\section{Período de avaliação}

O período de avaliação influenciou o tempo gasto em ruminação e de outras atividades (Tabela 3). Menor tempo em outras atividades e maior tempo de ruminação foram observados no terceiro período experimental, fase mais próxima à parição. As vacas apresentaram menor número de mastigadas e tempo de ruminação por bolo ruminal durante o segundo período de avaliação, com valores respectivamente de 48,76; 45,84 e 49,88 mastigadas e 57,87; 53,07 e 56,13 segundos por bolo ruminal para o primeiro, segundo e terceiro período.

Com o avançar da gestação, as vacas melhoraram a seletividade do material ingerido, uma vez que apresentaram menor deslocamento entre as estações alimentares no terceiro período de avaliação (Tabela 3), com valor de 1,03 passo estação alimentar $^{-1}$, mas permaneceram menos tempo em cada estação de pastejo (6,42 segundos), percorrendo assim, maior número de estações minuto ${ }^{-1}$ (10,01 estações) e consequentemente mais estações alimentares ao longo do dia (5029 estações). Durante o primeiro e segundo período, os animais permaneceram mais tempo em cada estação alimentar (10,43 e 9,49 segundos, respectivamente), aspecto que reduziu o número de estações de pastejo frequentadas durante o turno diurno (3095 e 3636 estações alimentares, respectivamente).

Apesar da aparente melhora na eficiência de pastejo com o avançar da gestação, a apreensão de forragem medida pela taxa de bocados e número de bocados dia $^{-1}$ não foi influenciada pelo período de avaliação $(P>0,05)$, ou seja, a ingestão de forragem não parece ter sido alterada, mas sim, a seletividade do material ingerido pelas vacas de corte.

\section{Discussão}

Vacas sem suplementação (Baixo) apresentaram maior tempo de pastejo (Tabela 2), demonstrando que o suplemento para suprir 100 ou $150 \%$ das exigências nutricionais durante o terço final de gestação interfere no consumo de forragem dos animais. Segundo Sniffen et al. (1993), a ingestão do alimento nos bovinos é regulada pelos mecanismos: psicogênico, que envolve a resposta animal a fatores inibidores ou estimuladores relacionados ao alimento e ao ambiente; o fisiológico, no qual o controle é feito pelo balanço nutricional da dieta e portanto relacionado com a manutenção do equilíbrio energético; e o físico, associado à capacidade de distensão do rúmen e ao teor de fibra em detergente neutro (FDN). Nesse sentido, maior tempo de pastejo a medida que a gestação avança pode ser reflexo da menor capacidade de ingestão em função da limitação de espaço físico para o rúmem, devido ao maior tamanho do feto próximo ao momento da parição. Esta teoria justifica o aumento no tempo de pastejo e redução do período de outras atividades das matrizes no terceiro período de avaliação, onde as matrizes além de pastejarem por mais tempo, aumentaram a eficiência na captura do pasto, uma vez que reduziram seu deslocamento entre as estações alimentares e consequentemente percorreram mais estações alimentares durante o dia em busca de forragem de maior qualidade (Tabela 3 ).

O menor tempo de pastejo do tratamento Alto está relacionado com o maior tempo de alimentação no cocho e maior tempo de outras atividades destas matrizes. Segundo Hodgson (1990), os ruminantes adaptam-se às diversas condições de alimentação, manejo e ambiente, modificando seus parâmetros de comportamento ingestivo para alcançar e manter determinado nível de consumo, compatível com suas exigências nutricionais. Desse modo, o tempo destinado ao pastejo foi reduzido a medida que as exigências nutricionais foram sendo atendidas. Esse comportamento fica mais evidente ao analizarmos as frequências de pastejo dos animais durante o período experimental (Figuras 1, 2 e 3). Vacas do tratamento Alto permanceram mais tempo sem executarem atividades de alimentação ou de ruminação, em função do suplemento concentrado, 
alimento mais palatável e com maior valor nutricional que a pastagem nativa (Tabela 1), fator que acabou influenciando o tempo de outras atividades deste tratamento. Resultados semelhantes foram relatados por Missio et al, (2010) e Goularte et al., (2011), que também observaram aumento no tempo destinado a outras atividades a medida que a participação de alimento concentrado na dieta aumenta.

As baixas frequências de pastejo até o horário da suplementação das vacas dos tratamentos Médio e Alto, mesmo em horários com temperatura mais amena. podem ser associadas ao baixo valor nutricional da pastajem nativa no período (Tabela 1), além da baixa palatabilidade e Altos teores de fibra e lignina da forragem que foram relatados por (Silveira et al., 2014), que limitam o consumo de pasto em ruminantes. As matrizes submetidas à restrição alimentar na gestação (Baixo) apresentaram maior frequência de pastejo ao longo do dia. Hodgson et al. (1994) afirmaram que o tempo medio de pastejo diário fica em torno de 8 horas, porém, pode atingir 16 horas diárias em casos extremos de baixa qualidade do pasto ou altas exigências dos animais e a injestão de suplementos pode complementar as demandas nutricionais a ponto de reduzir o tempo de pastejo (Lee et al., 2013). As vacas suplementadas para atingir 100 e $150 \%$ das exigências nutricionais concentraram seus pastejos após a suplementação, saindo dos tradicionais horários de pastejo nas horas mais frescas do dia.

O período despendido para a atividade de ruminação diúrno foi baixo, mas semelhante entre os tratamentos, e pode estar relacionado à maior ruminação no período noturno, uma vez que os bovinos possuem comportamento gregário e costumam apresentar maiores picos de ruminação e outras atividades durante a noite. As variáveis tempo de ruminação, número de mastigadas e tempo de ruminação por bolo ruminal foram semelhantes entre os tratamentos. Alterações nesses parâmetros são esperadas quando há grande diferença no consumo de fibra em detergente neutro entre os tratamentos, o que não aconteceu no presente trabalho em função da pequena participação da suplementação concentrada no consumo total de alimento dos animais. Missio et al. (2010) observou que com a inclusão de 79\% de concentrado na dieta houve redução no tempo destinado ao consumo de alimento, ruminação, número de mastigadas mericíclicas por bolo e consequentemente, aumento no tempo em descanso (outras atividades).

Atividades relacionadas a ruminação (tempo dia $^{-1}$, tempo e mastigadas por bolo ruminal) foram maiores no período próximo ao parto das vacas, o que pode estar associado a maior exigência nutricional e baixa capacidade de consumo de forragem da matriz em função do maior tamanho do feto. Maiores teores de lignina e fibra da pastagem nativa no primeiro período (mês de agosto), explicam maior tempo e número de mastigadas por bolo ruminal regurgitado. Martini et al. (2017) também verificaram aumento no tempo de ruminação por bolo alimentar quando o teor de fibra aumentou.

Além de influenciar o tempo de pastejo, o nível nutricional pré-parto das vacas afetou a eficiência de pastejo, e consequentemente o consumo de forragem diário. Braga et al., (2007) descreve eficiência de pastejo como a ingestão e desaparecimento da forragem no piquete, fator que está relacionado à inúmeras características comportamentais que remetem às atividades do pastejo. Os mesmos autores verificaram que o aumento na oferta de forragem no piquete promove redução da eficiência de pastejo dos animais, uma vez que permite ao animal selecionar melhor a parte da planta a ser ingerida, aumentando consequentemente a variabilidade espacial da vegetação da pastagem. Quando a disponibilidade o qualidade da forragem limita o consumo de alimento, os animais alteram o comportamento ingestivo, utilizando mecanismos como diminuição do tamanho de bocado ou aumento da taxa de bocados (Fischer, 1996).

A seleção de pastejo ficou mais evidente nos animais do Alto, os quais permaneceram menos tempo por estação alimentar (6,94 segundos), com deslocamento de apenas 1,14 passos entre elas, percorrendo consequentemente, maior número de estações alimentares por minuto. A seletividade da melhor parte da planta a ser ingerida fica justificada ainda, pelo maior tempo por bocado para os tratamentos Médio ou Alto em comparação com o Baixo. Vacas do PV também permaneceram mais tempo por estação alimentar, e menor tempo de bocado, o que indica menor seleção de forragem. Segundo Prache e Peyraud 
(2001), os animais são motivados a trocar de estação alimentar quando percebem a possibilidade de ingerir melhor qualidade de pasto, sendo atributo de animais que possuem menores necessidades imediatas de ingerir nutrientes.

A utilização do suplemento concentrado para atender 100 ou $150 \%$ das exigências de proteína e energia no terço final de gestação de vacas de corte, período que coincide com as piores condições forrageiras no sistema de produção, melhora a ingestão de nutrientes e consequentemente a disponibilidade de nutrientes para a formação fetal. Neste sentido, a suplementação altera o comportamento ingestivo dos animais, permitindo maior seletividade do pasto pelas vacas com maior nível de suplementação, o que de certa forma também promove e favorece o bem-estar-animal na fase de gestação onde as fêmeas possuem deslocamento dificultado pelo grande volume abdominal, uma vez que com a maior ingestão nutricional, estas podem permanecer mais tempo sem exercer nenhuma atividade pastoril.

Tabela 2 - Interação entre período e tratamento para tempo de pastejo de vacas submetidas à diferentes níveis nutricionais préparto.

\begin{tabular}{lcccc}
\hline Tratamentos & & Períodos & Médias \\
\cline { 2 - 4 } & P1 & P2 & P3 & 9,75 \\
\hline Baixo & $10,11^{\mathrm{a}}$ & $9,99^{\mathrm{a}}$ & $9,16^{\mathrm{ab}}$ & 8,37 \\
Médio & $7,77^{\mathrm{cd}}$ & $8,80^{\mathrm{b}}$ & $8,55^{\mathrm{bc}}$ & 6,95 \\
Alto & $6,55^{\mathrm{d}}$ & $6,63^{\mathrm{d}}$ & $7,66^{\mathrm{c}}$ & \\
Médias & 8,14 & 8,47 & 8,46 & \\
\hline
\end{tabular}

Médias seguidas por letras distintas na mesma linha diferem entre si pelo texto de tukey a $5 \%$ de probabilidade $(\mathrm{P}<0,05)$.

$\mathrm{P}=0,0052$; Erro padrão da média $= \pm 0,30$.

Fonte: Autores.

Figura 1 - Temperatura do ar e frequência de pastejo de vacas gestantes submetidas à diferentes níveis nutricionais no terço final de gestação ( $7^{\circ}$ mês de gestação).

a)

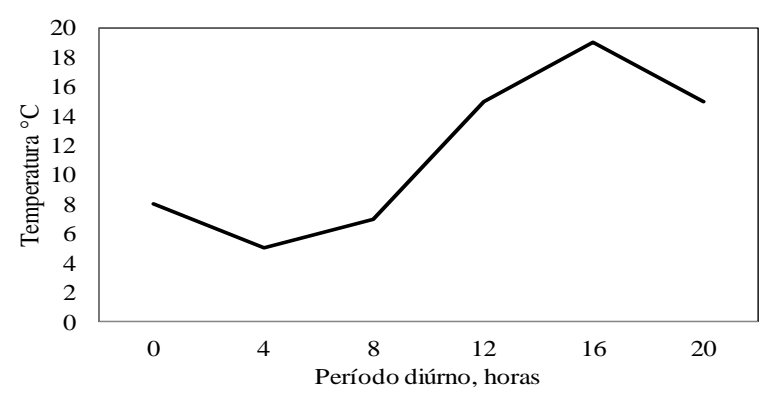

c)

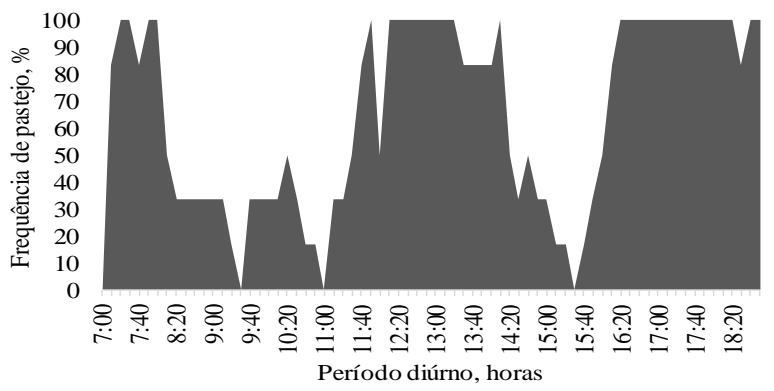

b)

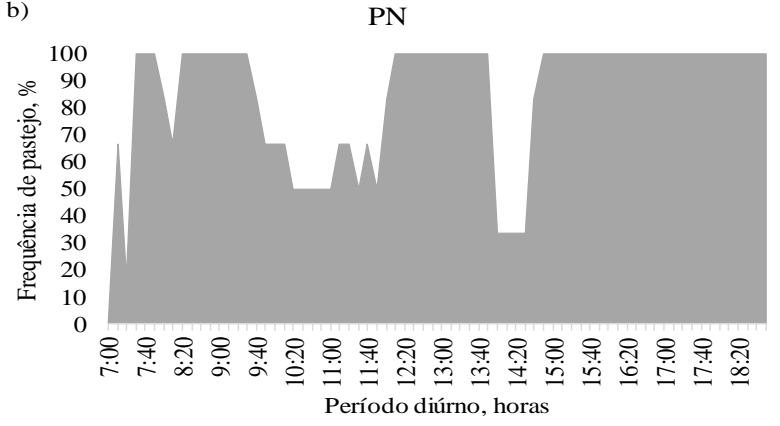

d)

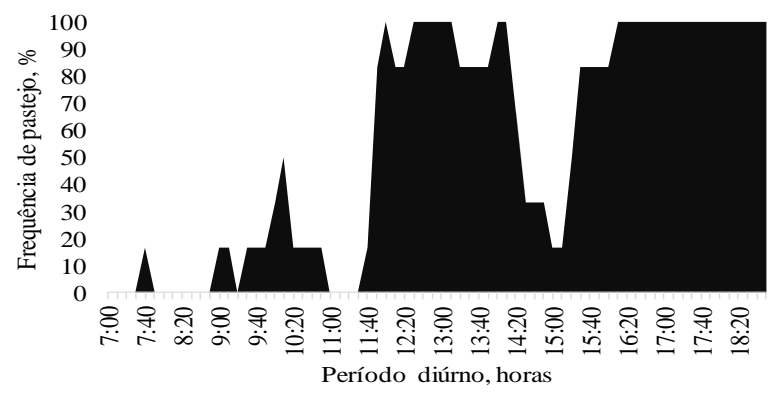

Fonte: Autores. 
Figura 2 - Temperatura do ar e frequência de pastejo de vacas gestantes submetidas à diferentes níveis nutricionais no terço final de gestação $\left(8^{\circ}\right.$ mês de gestação).

a)
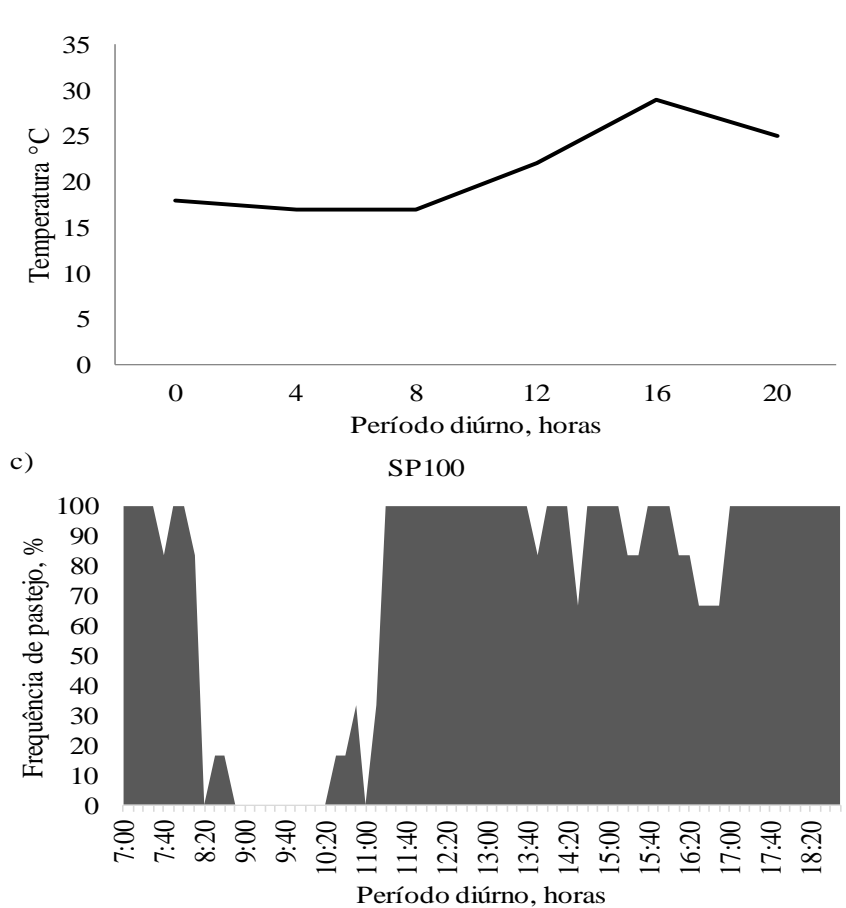

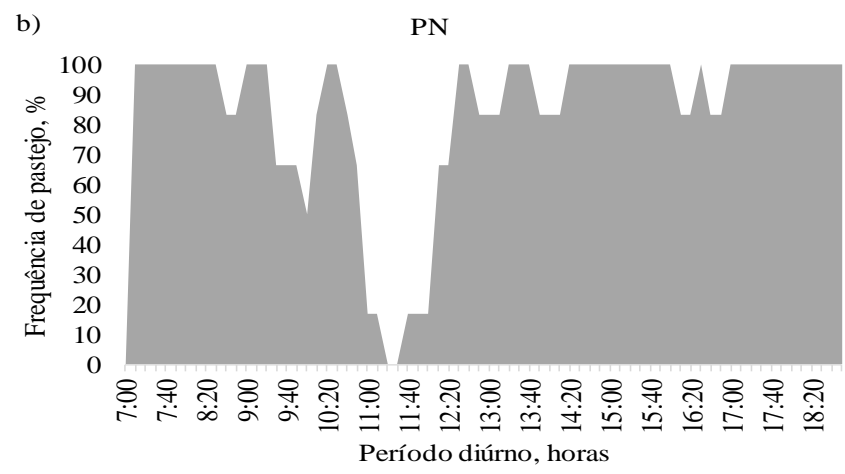

d)

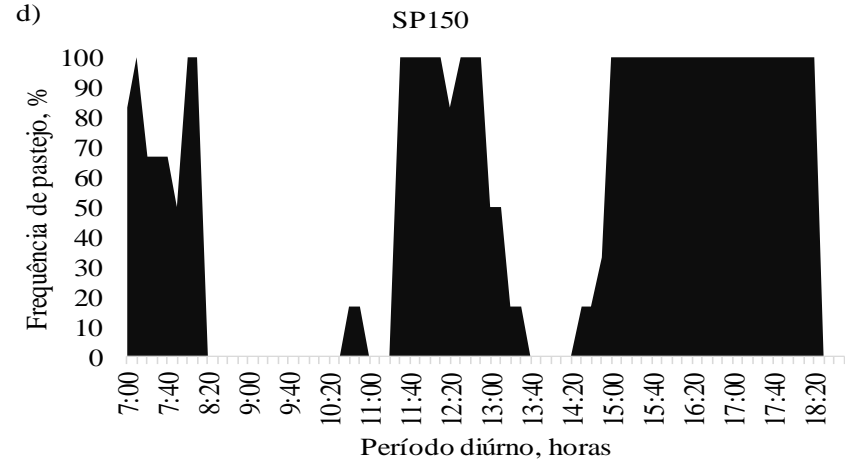

Fonte: Autores.

Figura 3 - Temperatura do ar e frequência de pastejo de vacas gestantes submetidas a diferentes níveis nutricionais no terço final de gestação $\left(9^{\circ}\right.$ mês de gestação).

a)

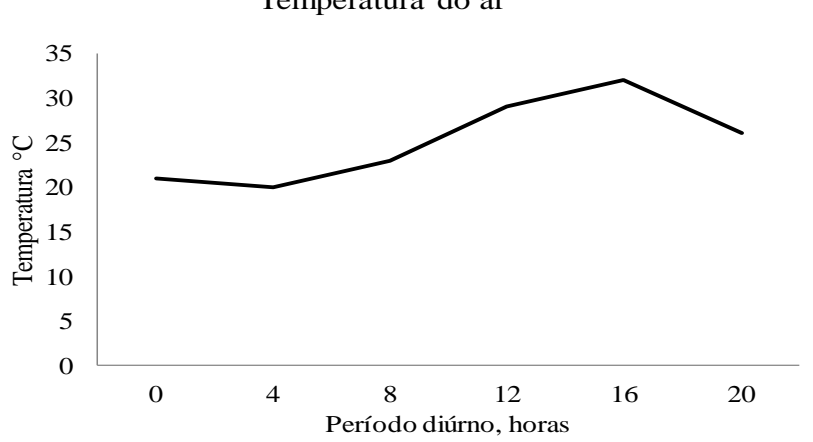

c)

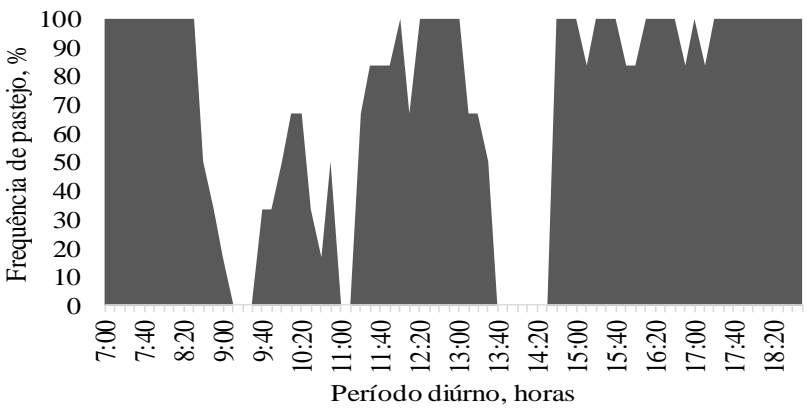

b)

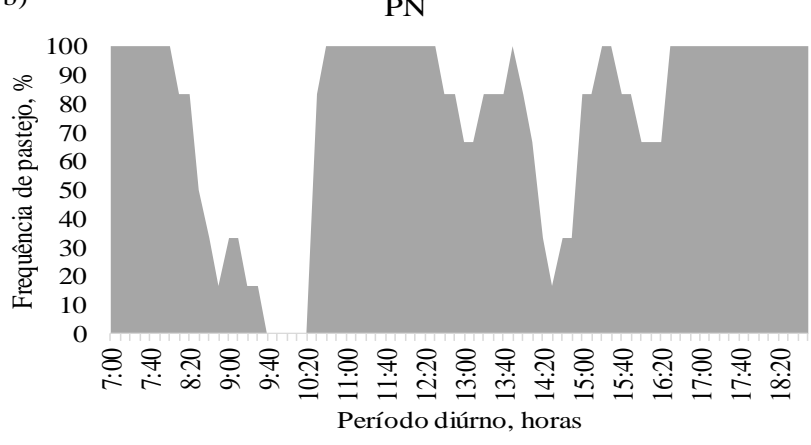

d)

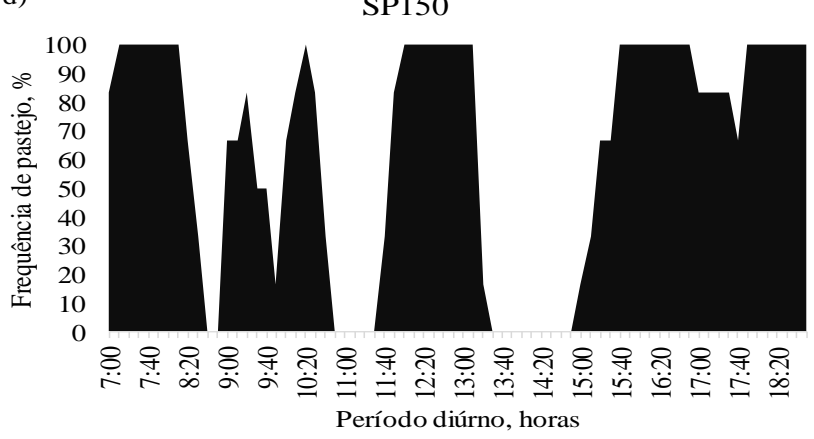


Research, Society and Development, v. 10, n. 7, e19810716555, 2021

(CC BY 4.0) | ISSN 2525-3409 | DOI: http://dx.doi.org/10.33448/rsd-v10i7.16555

Tabela 3 - Comportamento ingestivo de vacas gestantes de acordo com o nível nutricional pré-parto.

\begin{tabular}{|c|c|c|c|c|c|c|c|c|c|c|}
\hline \multirow{2}{*}{ Aspectos comportamentais } & \multicolumn{3}{|c|}{ Tratamentos } & \multirow{2}{*}{ P-valor } & \multirow{2}{*}{ EPM } & \multicolumn{3}{|c|}{ Períodos } & \multirow{2}{*}{ P-valor } & \multirow{2}{*}{ EPM } \\
\hline & Baixo & Médio & Alto & & & $\mathrm{P} 1$ & $\mathrm{P} 2$ & P3 & & \\
\hline Tempo de outras atividades, $\mathrm{h}$ & $0,96^{\mathrm{c}}$ & $1,86^{\mathrm{b}}$ & $3,24^{\mathrm{a}}$ & $<0,0001$ & 0,14 & $2,56^{\mathrm{a}}$ & $1,97^{\mathrm{ab}}$ & $1,54^{\mathrm{b}}$ & $<0,0003$ & 0,14 \\
\hline Tempo de ruminação, $h$ & 1,26 & 1,44 & 1,20 & 0,4346 & 0,13 & $0,97^{\mathrm{b}}$ & $1,27^{\mathrm{b}}$ & $1,66^{\mathrm{a}}$ & 0,0032 & 0,13 \\
\hline Mastigadas bolo ruminal $^{-1}$ & 46,85 & 49,02 & 48,60 & 0,7293 & 2,01 & $48,76^{\mathrm{a}}$ & $45,84^{\mathrm{b}}$ & $49,88^{\mathrm{a}}$ & 0,0413 & 1,49 \\
\hline Tempo de ruminação bolo ruminal ${ }^{-1}, \mathrm{~s}$ & 55,68 & 55,38 & 56,02 & 0,9799 & 2,24 & $57,87^{\mathrm{a}}$ & $53,07^{\mathrm{b}}$ & $56,13^{\mathrm{a}}$ & 0,0054 & 1,59 \\
\hline Passos minuto $^{-1}$ & 9,19 & 8,94 & 10,87 & 0,2255 & 0,79 & 8,14 & 10,47 & 10,40 & 0,0776 & 0,79 \\
\hline Passos dia ${ }^{-1}$ & 5408 & 4493 & 4528 & 0,1840 & 315,31 & $3868^{b}$ & $5327^{\mathrm{a}}$ & $5234^{\mathrm{a}}$ & 0,0093 & 409,73 \\
\hline Tempo estação alimentar ${ }^{-1}, \mathrm{~s}$ & $9,18^{\mathrm{a}}$ & $10,19^{\mathrm{a}}$ & $6,96^{\mathrm{b}}$ & 0,0180 & 0,70 & $10,43^{\mathrm{a}}$ & $9,49^{\mathrm{a}}$ & $6,42^{\mathrm{b}}$ & 0,0002 & 0,70 \\
\hline Estação alimentar minuto $^{-1}$ & $7,06^{\mathrm{b}}$ & $7,31^{\mathrm{b}}$ & $9,51^{\mathrm{a}}$ & 0,0160 & 0,53 & $6,51^{\mathrm{b}}$ & $7,35^{\mathrm{b}}$ & $10,01^{\mathrm{a}}$ & 0,0002 & 0,53 \\
\hline Estação alimentar dia ${ }^{-1}$ & 4115 & 3661 & 3984 & 0,4611 & 255,24 & $3095^{\mathrm{b}}$ & $3636^{\mathrm{b}}$ & $5029^{\mathrm{a}}$ & $<0,0001$ & 255,24 \\
\hline Tempo bocado ${ }^{-1}, \mathrm{~s}$ & $1,45^{\mathrm{b}}$ & $1,64^{\mathrm{a}}$ & $1,72^{\mathrm{a}}$ & 0,0081 & 0,04 & 1,65 & 1,61 & 1,55 & 0,3594 & 0,04 \\
\hline Bocados minuto $^{-1}$ & $41,86^{\mathrm{a}}$ & $37,37^{\mathrm{b}}$ & $35,39^{\mathrm{b}}$ & 0,0057 & 1,10 & 37,00 & 38,39 & 39,23 & 0,3622 & 1,10 \\
\hline Bocados dia ${ }^{-1}$ & $23119^{a}$ & $18693^{b}$ & $14811^{\mathrm{c}}$ & 0,0009 & 1062,65 & 18139 & 18498 & 19986 & 0,4377 & 1062,65 \\
\hline
\end{tabular}

Médias seguidas por letras distintas na mesma linha diferem entre si pelo texto de tukey a $5 \%$ de probabilidade $(\mathrm{P}<0,05)$;

$\mathrm{s}=$ segundos; $\mathrm{h}=$ horas.

Fonte: Autores. 


\section{Conclusão}

Vacas suplementadas para atender $150 \%$ das exigências nutricionais no terço final de gestação apresentam maior tempo de outras atividades, menor tempo por estação alimentar e percorrem maior número de estações de pastejo por minuto, enquanto que vacas sem suplementação apresentam menor tempo por bocado capturado, e realizam maior número de bocados ao longo do dia.

O avanço da gestação reduz o tempo de outras atividades e aumenta o tempo de ruminação das vacas. A proximidade do parto reduz o deslocamento e o tempo gasto por estação alimentar, e aumenta o número de estações de pastejo frequentadas ao longo do dia.

A suplementação complementar e o avanço da gestação melhoram a seletividade do pasto ingerido pelas vacas de corte, enquanto que a menor oferta de nutrientes aumenta a eficiência de pastejo dos animais.

\section{Referências}

Alvares, C. A., Stape, J. L., Sentelhas, P. C., Gonçalves, J. L. M., \& Sparovek, G. (2013). Köppen's climate classification map for Brazil. Meteorologische Zeitschrift, 22, 711-728. 10.1127/0941-2948/2013/0507

Barcellos, J. O. J. \& Oaigen, R. P. I. (2014). Cadeia produtiva da carne bovina e os sistemas de produção na bovinocultura de corte. In: Oaigen, R.O. (Coord.). Gestão na Bovinocultura de Corte. Guaíba: Agro livros, 21-41.

Bauman, D. E. \& Currie, B. (1980). Partitioning of nutrients during pregnancy and lactation: a review of mechanisms involving homeostasis e homeorhesis. Journal of Dairy Science, 63, 1514-1529. doi:10.3168/jds.S0022-0302(80)83111-0

Bohnert, D. W., Stalker, L. A., Nyman, A., Falck, S. J., \& Cooke, R. F. (2013). Late gestation suplementation of beff cows differing in body condition score: Effects on cow and calf performance. Journal of Animal Science, 91, 5485-5491. 10.2527 / jas.2013-6301

Braga, G. J., Pedreira, C. G. S., Herling, V. R., \& Luz, P. H. C. (2007). Eficiência de pastejo de capim-marandu submetido a diferentes ofertas de forragem. Pesquisa Agropecuária Brasileira, 42, 1641-1649. 10.1590/S0100-204X2007001100017

Du, M., Tong, J., Zhao, J., Underwood, K. R., Zhu, M., Ford, S. P., \& Nathanielsz, P. W. (2010). Fetal programming of skeletal muscle development in ruminant animals. Journal Animal Science, 88, 51-60. 10.2527 / jas.2009-2311

Goularte, S. R., et al. (2011). Comportamento ingestivo e digestibilidade de nutrientes em vacas submetidas a diferentes níveis de concentrado. Arquivo Brasileiro de Medicina Veterinária e Zootecnia, 63(2), 414-422. 10.1590/S0102-09352011000200020

Hodgson, J. (1982). Infuence of sward characteristics on diet selection and herbage intake by the grazing animal. In: HACKER, J.B. (ed.) Nutritional limits to animal production from pastures. Queensland, 153-166.

Hodgson, J. (1990). Grazing management: science into practice. Longman Handbooks in Agriculture, 203.

Hodgson, J., Clark, D. A., \& Mitchell, R. J. (1994). Foraging behavior in grazing animals and its impact on plant communities. In: FAHEY Jr, G.C. (Ed). Forage quality, evaluation and utilization. Nacional Conference on Forage Quality. American Society of Agronomy, $796-827$.

Laca, E. A., Ungar, E. D., Seligman, N. G., \& Demment, M. W. (1992). Effects of sward height and bulk density on bite dimensions of cattle grazing homogeneous swards. Grass and Forage Science, 47, 91-102. 10.1111/j.1365-2494.1992.tb02251.x

Lee, C., Fisher, A. D., Colditz, I. G., Lea, J. M., \& Ferguson, D. M. (2013). Preference of beef cattle for feedlot or pasture environments. Applied Animal Behaviour Science, 145, 53-59. 10.1016/j.applanim.2013.03.005

Martini, P. M., Brondani, I. L., Alves Filho, D. C., Viana, A. F. P., Adams, S. M., Klein, J. L., Rodrigues, L. S., \& Cattelam, J. (2017). Comportamento ingestivo de bovinos jovens mantidos em pasto de sorgo ou milheto. Boletim da Indústria Animal, 74, 308-318. 10.17523/bia.v74n4p308

Mello, J. C. C. B. S., Gomes, E. G., Abreu, U. G. P., Carvalho, T. B., \& Zen, S. (2013). Análise de desempenho de sistemas de produção modais de pecuária de cria no Brasil. Produção, 23(4), 877-886. 10.1590/S0103-65132013005000010

Missio, R. L., Brondani, I. L., Alves Filho, D. C., Silveira, M. F., Freitas, L. S., \& Restle, J. (2010). Comportamento ingestivo de tourinhos terminados em confinamento, alimentados com diferentes níveis de concentrado na dieta. Revista Brasileira de Zootecnia, 39, 1571-1578. 10.1590/S151635982010000700025

National Research Council. (1998). Nutrient requirements of beef cattle. Oklahoma State University: Division of Agricultural Sciences and Natural Resources.

Pereira, C. H., Patino, H. O., Hoshide, A. K., Abreu, D. C., Rotz, C. A., \& Nabinger, C. (2018). Grazing supplementation and crop diversification benefits for southern Brazil beef: A case study. Agricultural Systems, 62, 1-9. 10.1016/ j.agsy.2018.01.009

Prache, S. \& Peyraud, J. (2001). Foraging behavior and intake in temperate cultivated grassland. In: International Grassland Congress, $309-319$.

Sas Institute Inc. (2016). Statistical Analysis System. User’s guide version 3.5 SAS ${ }^{\circledR}$ Studio University Edition. Cary, North Carolina. 
Research, Society and Development, v. 10, n. 7, e19810716555, 2021

(CC BY 4.0) | ISSN 2525-3409 | DOI: http://dx.doi.org/10.33448/rsd-v10i7.16555

Silveira, M. F., et al. (2014). Suplementação com gordura protegida para vacas de corte desmamadas precocemente mantidas em pastagem natural. Arquivo Brasileiro de Medicina Veterinária e Zootecnia, 66, 809-817. 10.1590/1678-41625366

Sniffen, C. J., Beverly, R. W., Mooney, C. S. Roe, M. B., \& Skidmore, A. L. (1993). Nutrient requirement versus supply in dairy cow: Strategies to account for variability. Journal of Dairy Science, 76, 3160-3178. https://www.journalofdairyscience.org/article/S0022-0302(93)77655-9/pdf

Wilm, H. G., Costello, D. F., \& Klipple, G. E. (1944). Estimating forage yield by the double-sampling methods. Journal of American Society of Agronomy, 36, 194-203. 10.2134/agronj1944.00021962003600030003x 